\section{Altered parasite life-cycle processes characterize Babesia divergens infection in human sickle cell anemia}

\author{
Jeny R. Cursino-Santos, ${ }^{1}$ Manpreet Singh, ${ }^{1}$ Eric Senaldi, ${ }^{2}$ Deepa Manwani, ${ }^{3}$ \\ Karina Yazdanbakhsh ${ }^{4}$ and Cheryl A. Lobo ${ }^{1}$ \\ ${ }^{1}$ Department of Blood-Borne Parasites Lindsley F. Kimball Research Institute, New York

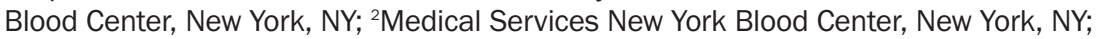 \\ ${ }^{3}$ Department of Pediatrics, Albert Einstein College of Medicine, Bronx, NY and \\ ${ }^{4}$ Department of Complement Biology Lindsley F. Kimball Research Institute, New York \\ Blood Center, New York, NY, USA
}

\section{ABSTRACT}

$B$ abesia divergens is an intra-erythrocytic parasite that causes malaria-like symptoms in infected people. As the erythrocyte provides the parasite with the infra-structure to grow and multiply, any perturbation to the cell should impact parasite viability. Support for this comes from the multitude of studies that have shown that the sickle trait has in fact been selected because of the protection it provides against a related Apicomplexan parasite, Plasmodium, that causes malaria. In this paper, we examine the impact of both the sickle cell anemia and sickle trait red blood cell (RBC) environment on different aspects of the $B$. divergens life-cycle, and reveal that multiple aspects of parasite biological processes are altered in the mutant sickle anemia RBC. Such processes include parasite population progression, caused potentially by defective merozoite infectivity and/or defective egress from the sickle cell, resulting in severely lowered parasitemia in these cells with sickle cell anemia. In contrast, the sickle trait $\mathrm{RBC}$ provide a supportive environment permitting in vitro infection rates comparable to those of wildtype $\mathrm{RBC}$. The elucidation of these naturally occurring $\mathrm{RBC}$ resistance mechanisms is needed to shed light on host-parasite interaction, lend evolutionary insights into these related blood-borne parasites, and to provide new insights into the development of therapies against this disease.

\section{Introduction}

The human erythrocyte serves as the common host cell for two major Apicomplexan parasites, Plasmodium and Babesia. Both species invade, develop and egress from the red blood cell (RBC) following specific developmental programs that contribute to the majority of clinical symptoms associated with these infections. ${ }^{1-3}$ Shielded within the host RBC, these intra-erythrocytic parasites differentiate and multiply while concealing their presence from the immune system. After filling the available intra-cellular space, the resultant parasite progeny egress as merozoites, and invade new RBC, carrying on the cycle of growth and proliferation. This cycle depends on intricate interactions between host RBC and parasite molecules. Thus, any perturbations to either the composition or arrangement of proteins on or within the host RBC can impact the parasite's development and survival, and thus increase host resistance to parasite infection.

Much effort has been spent in determining the different means by which the human host can suppress this active parasite replication to limit the damage caused by the continuous destruction of the host RBC. One of the most commonly encountered mechanisms discovered is the genetic disorders that are found in the RBC. ${ }^{4}$ As the Plasmodium parasites have long co-existed with the human host, they have exerted extraordinary adaptive pressure on the human species. ${ }^{5}$ Consequently, in humans, multiple genetic polymorphisms have been selected for several hemoglobin disorders that provide intrinsic protection against severe malaria complications and are convincingly supported by clinical data. ${ }^{6,7}$ Hemoglobin $(\mathrm{Hb})$ is the oxy-
Ferrata Storti Foundation
Haematologica 2019

Volume 104(11):2189-2199

\section{Correspondence:}

CHERYL A. LOBO

CLobo@NYBloodcenter.org

Received: December 13, 2018.

Accepted: March 20, 2019

Pre-published: March 28, 2019

doi:10.3324/haematol.2018.214304

Check the online version for the most updated information on this article, online supplements, and information on authorship \& disclosures: www.haematologica.org/content/104/11/2189

\section{(C)2019 Ferrata Storti Foundation}

Material published in Haematologica is covered by copyright. All rights are reserved to the Ferrata Storti Foundation. Use of published material is allowed under the following terms and conditions:

https://creativecommons.org/licenses/by-nc/4.0/legalcode. Copies of published material are allowed for personal or internal use. Sharing published material for non-commercial purposes is subject to the following conditions: https://creativecommons.org/licenses/by-nc/4.0/legalcode, sect. 3. Reproducing and sharing published material for commercial purposes is not allowed without permission in writing from the publisher. 
gen-carrying component and major protein of the RBC and is normally formed as a tetramer of two $\alpha$-globins and two $\beta$-globins which constitute adult hemoglobin $A$ $(\mathrm{HbA})$. The major hemoglobinopathies result from mutations that either decrease the production of $\alpha$ - or $\beta$-globins (in $\alpha$ - and $\beta$-thalassemia) or sickling of the erythrocyte (in sickle $\mathrm{HbS}, \mathrm{HbC}$, and $\mathrm{HbE}$ diseases)., Remarkably, small genetic variations confer dramatic levels of protection from malaria. ${ }^{10,11} \mathrm{HbS}$ is the result of a single point mutation $(\mathrm{Glu} \rightarrow \mathrm{Val})$ on the sixth codon of the $\beta$-globin gene. Homozygotes for hemoglobin S (HbSS) with two affected $\beta$ chains develop sickle cell disease (SCD), in which polymerized $\mathrm{Hb}$ causes $\mathrm{RBC}$ to sickle and occlude blood vessels, and results in high morbidity and mortality. ${ }^{12}$ Heterozygotes for sickle hemoglobin (HbAS) have sickle cell trait and are generally asymptomatic. Despite the obvious deleterious nature of $\mathrm{HbSS}$, it is now widely accepted that the persistence of the sickle mutation in human populations is due to the protection from malaria afforded to heterozygous individuals. ${ }^{13,14}$ Multiple divergent mechanisms have been put forward to explain this resistance to malaria, including enhanced macrophage uptake, impaired growth and maturation of parasite, and decreased deposition of parasitized RBC in deep post capillary beds, but no single convincing explanation has yet been given. ${ }^{1,15,16}$

Babesiosis has long been recognized as a veterinary problem of great significance, but only in the last 50 years has it been recognized as an important pathogen in man. ${ }^{2}$ The four identified Babesia species that have so far been definitively confirmed to infect humans are $B$. microti, ${ }^{17} B$. divergens, ${ }^{18} B$. duncani, ${ }^{19,20}$ and $B$. venatorum. ${ }^{21-24}$ As sampling has become expansive and techniques have become more sensitive, there is evidence that more $B$. microti-like and $B$. divergens-like spp. are able to cause human infection (as reported in detail by Yabsley and Shock). ${ }^{25}$ However, the general life cycle within humans remains the same. Babesia parasites are intracellular obligates that target $\mathrm{RBC}$, and the parasite's ability to first recognize and then invade host $\mathrm{RBC}$ is central to the disease pathology. Besides its natural route of transmission via the infected tick, the parasite is also transmitted by transfusion of infected blood as its RBC host provides an optimum vehicle to facilitate its transmission. In fact, as the frequency of clinical cases has risen, there has been an associated increase in transfusion-transmitted Babesia (TTB), mainly reported for $B$. microti, ${ }^{26-28}$ making babesiosis the most frequent transfusion-transmitted infection in the US. Patients with sickle cell anemia, especially those on chronic transfusion therapy, are at high risk for severe TTB. ${ }^{29,30}$ Whether the sickle red cells themselves are responsible for the increased susceptibility of these patients to TTB or whether this is due to other related factors, such as a compromised immune system, has not been investigated. In this paper, we focus on the ability of the Babesia parasite to invade, grow in and egress from sickle trait and sickle cell anemia erythrocytes. Use of in vitro invasion and development assays were developed in our laboratory, ${ }^{31}$ as our primary outcome provided a rare opportunity to systematically examine the cellular determinants of parasite development in the sickle cell anemia setting. These enabled a comparison between various components of the parasite life-cycle in RBC obtained from various hemoglobin genotypes, $\mathrm{HbAA}, \mathrm{HbAS}$ and $\mathrm{HbSS}$, and revealed altered parasite population progres- sion, parasite maturation and egress phenotypes in the HbSS cells.

\section{Methods}

\section{Ethics statement}

Human blood from healthy volunteer donors was used to culture $B$. divergens $(\mathrm{Bd})$ in vitro. SCD patients' $\mathrm{RBC}$ were obtained from residual anticoagulated blood samples from same day collections from patients with sickle cell anemia (hemoglobin genotype SS) who had not been transfused for at least three months prior to sample draw. Patients provided consent for use of de-identified blood for research purposes on a Montefiore Medical Center Institutional Review Board (IRB) approved protocol. HbAA RBC and sickle trait RBC were identified from New York Blood Center (NYBC) blood donors and confirmed through genotypic analysis. All blood was used within a few hours of drawing. The blood was de-identified and approved for use by the NYBC IRB. All blood donors gave informed written consent for use of their blood for research purposes.

\section{B. divergens in vitro culture}

$B$. divergens (Bd Rouen 1986 strain) were maintained in human $\mathrm{RBC}$ as previously described. ${ }^{32,33} \mathrm{~A}^{+} \mathrm{RBC}$ were collected in $10 \%$ CPD and washed 3X with RPMI 1640 medium for the complete plasma and white cell removal.

\section{Free merozoites isolation}

High concentration of viable free merozoites was isolated from unsynchronized cultures at high parasitemia (40\%), as described previously. ${ }^{31,34}$

\section{Assessment of invasion, development and egress in various red blood cells}

Fresh cultures were seeded with purified merozoite suspension at $20 \%(\mathrm{v} / \mathrm{v})$ of culture volume. To define time points to accurately estimate invasion in the different RBC (HbAA, HbAS; HbSS), invasion was assayed in the first set of samples at 5 minutes (min), 1 hour (h) or $6 \mathrm{~h}$ post invasion. At additional time points (24-72 h), samples were collected to assess the culture progression and subpopulation dynamics from the perspective of parasite development and egress. Analysis was carried out at specific intervals of $24 \mathrm{~h}, 48 \mathrm{~h}$ and $72 \mathrm{~h}$ for the majority of cultures ( 6 cultures were monitored for $48 \mathrm{~h}$ ). The culture size (parasitemia) and the parasite proliferation analysis were carried out by FACS (described below). Characterization of parasite morphology and development was performed by light microscopy. Cells were obtained from three replicate cultures for each RBC sample.

\section{Light microscopy}

Blood smears were fixed with methanol and stained with $20 \%$ Giemsa (Sigma-Aldrich, St. Louis, MO, USA) for the morphological analysis of parasites. A minimum of 2000 cells was scanned for assessment of changes in morphology using a Nikon Eclipse E 600 microscope.

\section{Flow cytometry}

The dual-color staining protocol was used to monitor the parasite cycle within the RBC over $72 \mathrm{~h}$, as previously described ${ }^{31}$ with modifications.

\section{Statistical analysis}

Parasitemia was defined as the total number of infected $\mathrm{RBC}$ (iRBC) in every $100 \mathrm{RBC}$, not taking into consideration the number of parasites seen in a given cell when measured by flow 
cytometry. Sub-populations were identified as a function of the presence and their number of intra erythrocyte parasites / genome (intra erythrocyte parasite load) where " $1 \mathrm{~N}$ " refers to one genome copy based on the method previously describe. ${ }^{34}$ The percentage of inhibition mediated by the different $\mathrm{Hb}$ genotypes was determined by assuming that the parasitemia from control HbAA cells was equivalent to $0 \%$ inhibition or enhancement.

\section{Results}

\section{Response of $B$. divergens to the different host cell hemoglobin environments}

To assess the hosting ability of the sickle cell anemia cells, purified merozoite preparations were used to infect $\mathrm{HbSS}$ and HbAS RBC from sickle and sickle trait patients, respectively, in parallel with $\mathrm{HbAA} \mathrm{RBC}$ as wild-type control. Multiple independent experiments were performed with each cell type and processes such as invasion, development and egress were chronologically observed during the asexual erythrocytic cycle of $B$. divergens.

The first indication that there were differences in parasite development in the RBC of different $\mathrm{Hb}$ genotypes was observed while defining the ideal time points to evaluate various aspects of the parasite life-cycle (Table 1). A single sample each of HbAA, HbAS and HbSS RBC was used in an invasion assay using purified merozoite preparations. One-hour post-mixing was found to be the ideal time point to evaluate invasion and monitor the initial parasitemia. At the 5 min time point, the parasitemia was too low to assess differences between the three cell types, although high synchronicity of parasites was achieved. At the $6 \mathrm{~h}$ time point, the initial synchronicity was lost by events of late merozoite invasion and the cultures were affected by the long exposure to the ruptured RBC remnants that were introduced by the inoculation (data not shown). Because of these factors, $1 \mathrm{~h}$ post- mixing of merozoite preparations and RBC was used as the time-point to assess invasion in the experiments that followed.

$B$. divergens was able to efficiently invade $\mathrm{RBC}$ of all three genotypes: HbAA, HbAS and HbSS. However, parasite culture expansion was drastically compromised in the sickle cell (SS) environment which can be seen by the comparison of parasitemia progression in the HbSS culture as compared to both $\mathrm{HbAS}$ and $\mathrm{HbAA}$ cultures (Table 1) (culture growth inhibition of $36 \%, 59 \%$ and $70 \%$ respectively at $24 \mathrm{~h}, 48 \mathrm{~h}$ and $72 \mathrm{~h}$ post invasion of the HbSS culture when compared to $\mathrm{HbAA}$ ) (Figure1A). In contrast to the HbSS cultures, $\mathrm{HbAS}$ cells were found to provide a favorable environment for parasite progression, with only modest differences in parasitemia observed when compared with the wild-type HbAA (culture growth inhibition of $4.3 \%, 0 \%$ and $1 \%$ respectively at $24 \mathrm{~h}, 48 \mathrm{~h}$ and $72 \mathrm{~h}$ post invasion of HbAS culture compared to $\mathrm{HbAA}$ ) (Table 1 and Figure 1A).

\section{HbSS red blood cells from diverse donors exhibit comparable degrees of impairment of parasitemia progression while HbAS red blood cells from diverse donors permit normal parasite progression}

To assess the effect of the individual variability in sickle cell anemia and sickle trait RBC from different individuals on the parasite response to these $\mathrm{RBC}$ environments, $\mathrm{RBC}$ from 11 different sickle cell (Table 2) and five sickle trait (Table 3) patients were infected with purified merozoite preparations. Each experiment was run together with several wild-type RBC controls ( $\mathrm{HbAA}$ ) originating from different individuals. The cultures were monitored from invasion to $48-72 \mathrm{~h}$ post invasion.

On monitoring the parasite population sizes over time, it was clear that, although there was individual variation among sickle cells from different sickle cell anemia patients, the HbSS cells in every tested culture did not support normal culture progression, as the parasitemia was drastically reduced in all 11 sickle cell cultures (Table 2). This inhibition of HbSS-culture expansion was statistically significant at 48 h $(P<0.05)$, when HbAA RBC controls exhibited a robust approximately 3-4-fold increase in parasitemia, compared to the HbSS cells which remained static, exhibiting parasitemia close to the initial starting values. Eight HbSS cultures continued to be monitored at $72 \mathrm{~h}$ post invasion, to confirm if significant inhibition $(P<0.05)$ was maintained. Inhibition was found to be sustained and, once again, all eight cultures showed a plateau in parasitemia, not appreciably changed from their $48 \mathrm{~h}$ parasitemia, unlike the HbAA cultures which approach $40-50 \%$ parasitemia at 72 $\mathrm{h}$ (Table 2). From the perspective of parasitemia progression, there was thus an increased inhibition over time; approximately $73-92 \%$ inhibition of growth in HbSS cultures when compared to HbAA controls at $72 \mathrm{~h}$ (Figure 1B). In contrast, all HbAS cultures were able to reach a similar parasite population size as the control HbAA cultures at 72 $\mathrm{h}$, averaging $45 \%$ parasitemia, with no significant variations in parasitemia in individual $\mathrm{HbAS}$ donor cells at different time points $(P>0.05)$ (Table 3). Overall, all parasite cultures established in the HbAS-cells exhibited a similar increase in parasitemia when compared to the HBAA control (Tables 1 and 3), indicating the parasites capability for population progression in $\mathrm{HbAS} \mathrm{RBC}$, unlike the impaired culture growth phenotype observed in HbSS RBC. Table 4 shows the aggregate values of inhibition of parasite population progression in the HbSS cells and HbAS cells with reference to the parasitemias in HbAA cells at the three time points tested. Despite the HbSS cells and HbAS originating from diverse donors, the inhibition seen in the HbSS population is significantly higher than that seen in the $\mathrm{Hb}$ AS population which has close to parasitemia values in the HbAA population, at the $48 \mathrm{~h}$ (68\% inhibition in HbSS vs. $15 \%$ in $\mathrm{HbAS})$ and $72 \mathrm{~h}(84 \%$ inhibition in $\mathrm{HbSS}$ vs. $4 \%$ in $\mathrm{HbAS}$ ) time points in culture $(P<0.05)$ (Table 4$)$.

Table 1. Parasitemia assessed from cultures grown in single samples of each hemoglobin ( $\mathrm{Hb})$ genotype-HbAA, HbSS, HbAS cultures $(5$ min- 6 hours post inoculation of cultures with free merozoites) and at 24-hour intervals after inoculation (24-72 hours).

\begin{tabular}{|c|c|c|c|c|c|c|}
\hline Sample & $5 \mathrm{~min}$ & $1 \mathrm{~h}$ & $6 \mathrm{~h}$ & $24 \mathrm{~h}$ & 48 h & $72 \mathrm{~h}$ \\
\hline $\mathrm{HbAA}$ & $0.6[0.03]$ & $2.1[0.03]$ & $3.0[0.06]$ & $4.7[0.00]$ & $14.4[0.25]$ & $43.2[0.15]$ \\
\hline $\mathrm{HbSS}$ & $0.9[0.01]$ & $2.7[0.02]$ & $3.1[0.06]$ & $3.0[0.00]$ & $5.9[0.06]$ & $12.8[0.10]$ \\
\hline $\mathrm{HbAS}$ & $0.4[0.04]$ & $2.8[0.02]$ & $3.3[0.00]$ & $4.5[0.00]$ & $15.1[0.12]$ & $42.9[0.32]$ \\
\hline
\end{tabular}

Parasitemia values represent average percentage. [StDv]: Standard Deviation among triplicates; h: hours; min: minutes. 
Similar invasion efficiencies were obtained in HbAA, HbAS and HbSS red blood cells

To understand the specific defect induced in the parasite life-cycle by the sickle environment, the population structure of cultures was analyzed based on the parasite load within the infected RBC (number of parasite genomes by FACS analysis). By monitoring the distribution of sub-populations of infected RBC, the life cycle processes of invasion, parasite development and egress were evaluated in sickle cell anemia compared with sickle trait and control cells.

The invasion of merozoites in all cell types was measured by the percent parasitemia in the different cultures at the $1 \mathrm{~h}$ time point, established earlier to be the optimum point to assess invasion efficiency. The sickle RBC were able to support efficient merozoite invasion (HbSS-1 h parasitemia ranged from $0.8 \%$ to $2.3 \%$ ) (Tables 1 and 2 ). When the invasion efficiency of HbSS RBC was compared to that of control HbAA RBC, no significant difference in parasite invasion was observed among cultures $(P>0.05)$. In fact, the subtle increase in initial infection rates of HbSS versus $\mathrm{HbAA} \mathrm{RBC}$ was found to be an artefactual consequence of the presence of Howell-Jolly bodies (H-JB) in HbSS cells. These bodies, known as micronuclei, contain small erythrocyte DNA inclusions (approx. $1 \mu \mathrm{m}$ ) that are the consequence of cytogenetic damage. ${ }^{35,36}$ To confirm that the higher DNA content in infected HbSS cells was indeed from $\mathrm{H}-\mathrm{J}$ bodies, their quantification was first performed by their detailed counts on Giemsa stained smears of uninfected HbSS cells, followed by quantification of DNA content in uninfected HbSS cells (FACS analysis with Vybrant ${ }^{\circledR}$ DyeCycleTMGreen) (Figure 2). Our estimates of these bodies in all HbSS samples were in the range of $0.1-0.3 \%$ (shown for 3 independent HbSS samples in Online Supplementary Table S1), which when subtracted from the parasitemias found for the same infected
A

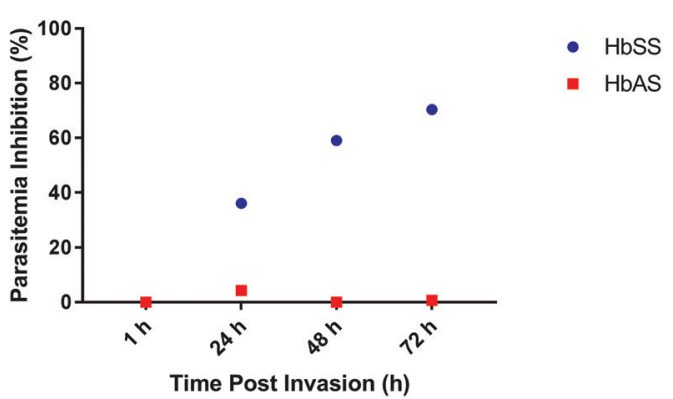

B

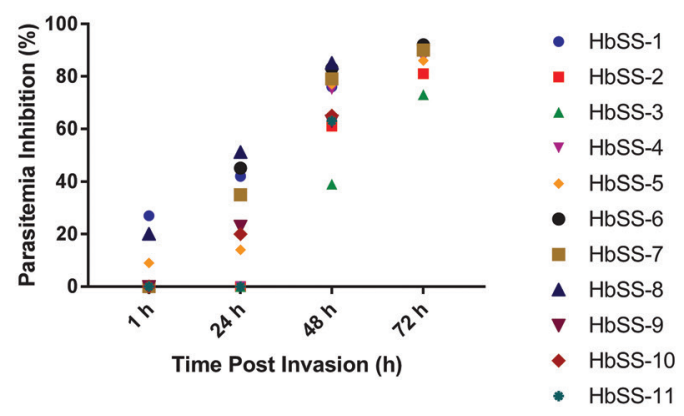

Figure 1. The expansion of $B$. divergens population was inhibited in HbSS cultures. Comparison of the growth inhibition rate between parasite cultures grown in $\mathrm{HbSS}$ cells and HbAS cells relative to growth in wild-type HbAA as measured by parasitemia. (A) Between cultures originating from a single HbSS and single $\mathrm{HbAS}$ donor. Values calculated based on data from Table 1. (B) Among HbSS cells from 11 different sickle cell disease patients, showing that, despite variation in parasitemia in individual cultures, all exhibit high degrees of inhibition of culture progression. Values calculated based on data from Table 2.

Table 2. Parasitemia of $B$. divergens cultures measured 1-72 hours post-invasion in sickle (HbSS) and wild-type (HbAA) red blood cells from different donors.

\begin{tabular}{lcccc}
\hline Sample* & $1 \mathrm{~h}$ & $24 \mathrm{~h}$ & $\mathbf{4 8} \mathrm{h}^{*}$ & $72 \mathrm{~h}^{*}$ \\
HbAA-I & $1.1[0.01]$ & $4.0[0.40]$ & $14.5[0.35]$ & $37.3[1.35]$ \\
HbSS-1 & $0.8[0.03]$ & $2.3[0.21]$ & $3.4[0.12]$ & $3.9[0.23]$ \\
\hline HbSS-2 & $1.5[0.01]$ & $4.5[1.18]$ & $5.6[0.12]$ & $7.2[0.21]$ \\
HbSS-3 & $1.9[0.02]$ & $5.1[0.84]$ & $8.9[0.38]$ & $9.9[0.10]$ \\
\hline HbAA-II & $1.1[0.03]$ & $4.1[1.06]$ & $20[0.92]$ & $43.4[0.21]$ \\
HbSS-4 & $2.0[0.02]$ & $4.6[1.56]$ & $5.1[0.07]$ & $5.5[0.28]$ \\
\hline HbSS-5 & $1.0[0.02]$ & $3.5[1.13]$ & $4.8[0.07]$ & $5.9[0.00]$ \\
HbAA-III & $1.9[0.02]$ & $5.5[0.26]$ & $21[0.15]$ & $49.3[0.75]$ \\
\hline HbSS-6 & $2.3[0.01]$ & $3.0[0.00]$ & $3.5[0.14]$ & $4.1[0.07]$ \\
HbSS-7 & $2.0[0.03]$ & $3.6[0.06]$ & $4.5[0.20]$ & $4.9[0.12]$ \\
\hline HbAA-IV & $1.0[0.03]$ & $2.9[0.06]$ & $12[0.25]$ & ND \\
HbSS-8 & $0.8[0.03]$ & $1.4[0.10]$ & $4.7[0.12]$ & ND \\
\hline HbSS-9 & $1.5[0.02]$ & $2.2[0.07]$ & $4.3[0.03]$ & ND \\
HbSS-10 & $1.5[0.04]$ & $2.3[0.02]$ & $4.2[0.15]$ & ND \\
\hline HbSS-11 & $1.5[0.01]$ & $3.0[0.35]$ & ND
\end{tabular}

*Four independent experiments. (HbSS 1-11) sickle samples (HbAA I-IV) wild-type control samples. Parasitemia values represent average percentage. [StDv]:Standard Deviation among triplicates. "Significant difference between wild-type controls HbAA and tested HbSS samples. $P<0.05$. ND: not determined; h: hours. 
HbSS cells, results in equivalent invasion efficiencies for $\mathrm{HbSS}$ and HbAA cells. Additionally, all the parasite cultures were followed a few hours later to ensure that parasite development could be recorded to yield comparable parasitemia. Images of invaded parasites can be very easily discriminated from that of the $\mathrm{H}-\mathrm{J}$ bodies, with the parasite seen as a distinct ring versus the solid staining pattern of the body (Figure 2). Overall, all cells were found to support parasite invasion equally well.

\section{Effect of sickle $\mathrm{Hb}$ (HbSS) and sickle trait $\mathrm{Hb}$ (HbAS) on the intra-erythrocytic parasite development}

The relative differences in parasitemia in the cultures (Tables 1-3) and in the distribution of their infected RBC sub-populations (Figure 3) described not only parasite competence for population expansion in that cell type, but also their developmental and differentiation status when monitoring the chronological emergence of each sub-parasite population within the infected RBC. The supplementary histogram peaks detected at $24 \mathrm{~h}$ post invasion (Figure 3) indicating parasite proliferation showed that the HbSS sickle cells, permitted intra-erythrocytic proliferation and differentiation of the parasites; however, they may not be providing an optimum environment for culture propagation as there was no appreciable increase in parasitemia after $24 \mathrm{~h}$.

As mentioned before, at $1 \mathrm{~h}$ post invasion $(1 \mathrm{~h}$ histograms) (Figure 3), all cultures exhibited similar FACS profiles, with the $1 \mathrm{~N}$ population seen as the dominant sub-population as expected, as shown in the representative samples. The distribution over time of these sub-populations of $\mathrm{iRBC}$ (frequency of events acquired in the histogram peaks corresponding to $1 \mathrm{~N}-$; $2 \mathrm{~N}-$-; $4 \mathrm{~N}-$; and $>4 \mathrm{~N}$ iRBC sub-populations), when translated into the line charts as presented in Online Supplementary Figure S1, showed that the conventional pattern of population formation was adopted by $B$. divergens in all RBC, irrespective of genotype, during the first $24 \mathrm{~h}$. During this first intraerythrocytic cycle, with the intense intra-cellular proliferation (characterized by the emergence of infected RBC with higher intra-cellular parasite load) and the low ratio of invasion/egress (as evidenced by the relatively stable culture sizes over $24 \mathrm{~h}$ as expected, even for the control cultures) Babesia similarly built its populations in all three cell types, exhibiting the common gain of heterogeneity by the presence of different sub-populations of iRBC.
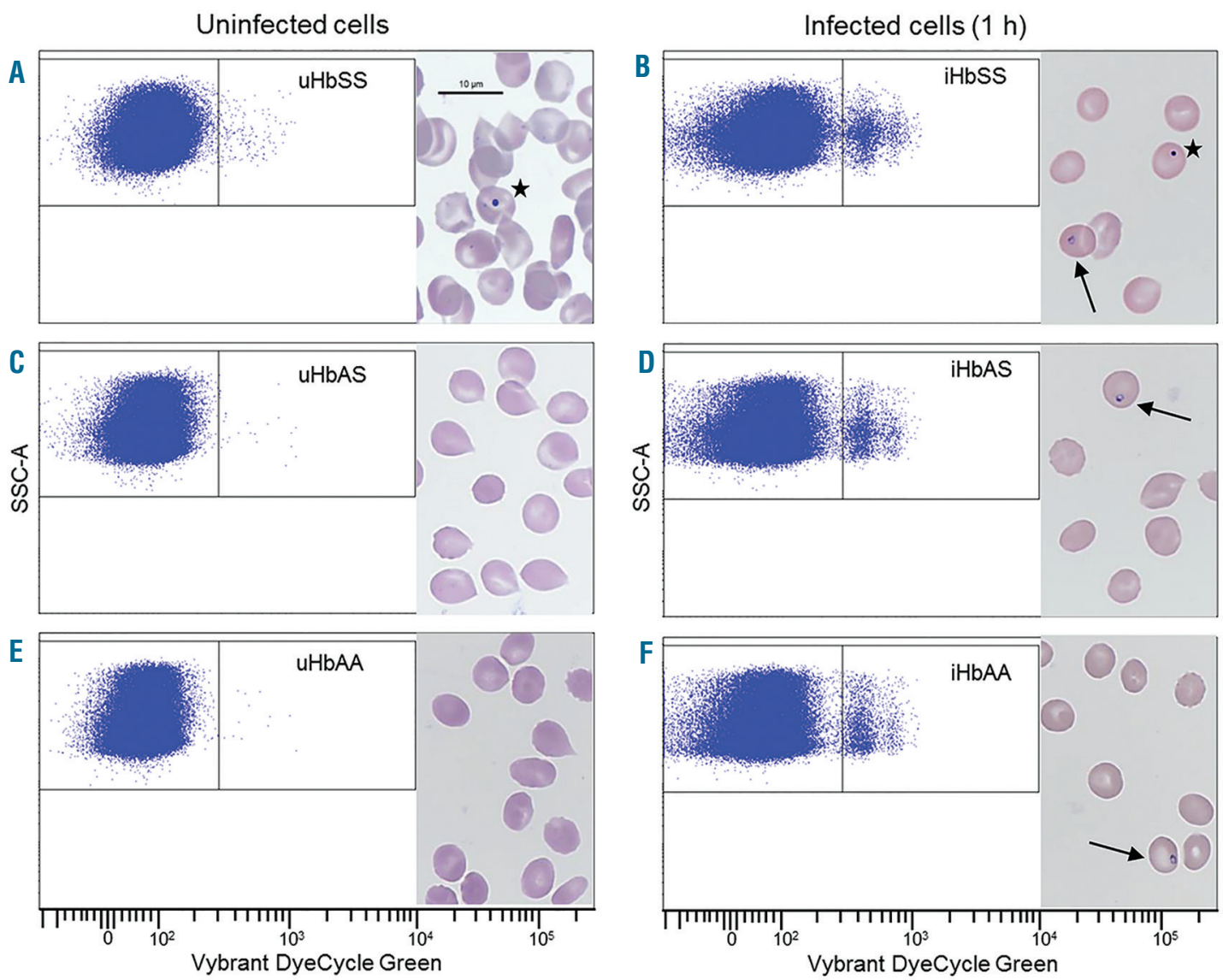

Figure 2. Invasion efficiency is similar in HbAA, HbAS and HbSS cells. FACS analysis of DNA content in representative (A,C,E) uninfected red blood cells (RBC) and (B,D,F) infected RBC, along with Giemsa stained smears. (A) HbSS cells reveal DNA positive population (0.1-0.3\%) from Howell Jolly (H-J) Bodies in cells, Giemsa image shows a dense $\mathrm{H}-\mathrm{J}$ body, (marked by asterisk). (C) HbAS cells and (E) HbAA cells do not have DNA positive population and Giemsa smear shows absence of bodies. (B) Parasite cultures in the same HbSS cells show higher Vybrant positive population than uninfected, being composed of both cells containing $\mathrm{H}$-J bodies and parasites. The difference between panels (B) and (A) yields invasion parasitemias (see Table 4 for data). Giemsa smear shows distinct ring formed 1 hour ( $\mathrm{h}$ ) post invasion, presenting a clear difference from $\mathrm{H}-\mathrm{J}$ body. ( $\mathrm{D}$ and $\mathrm{F}$ ) $1 \mathrm{~h}$ post-invasion cultures of (D) HbAS cells and (F) HbAA cells showing Vybrant positive population, and $1 \mathrm{~h}$ post-invasion Giemsa image showing newly invaded parasites (marked by arrows). 
Table 3. Parasitemia of $B$. divergens cultures measured 1-72 hours post invasion in sickle trait (HbAS) and wild-type (HbAA) red blood cells from different donors.

\begin{tabular}{lcccc} 
Sample & $\mathbf{1} \mathbf{h}$ & $\mathbf{2 4} \mathbf{h}$ & $\mathbf{4 8} \mathbf{h}$ & $\mathbf{7 2} \mathbf{h}$ \\
HbAA-V & $4.5[0.50]$ & $8.7[0.15]$ & $26.7[0.82]$ & $43.1[2.35]$ \\
HbAA-VI & $3.8[0.22]$ & $7.7[0.15]$ & $26.7[0.38]$ & $47.2[1.25]$ \\
\hline HbAA-VII & $3.3[0.12]$ & $7.6[0.21]$ & $26.0[0.26]$ & $48.2[0.70]$ \\
HbAS-1 & $2.8[0.04]$ & $6.7[0.23]$ & $23.8[1.01]$ & $41.8[3.35]$ \\
\hline HbAS-2 & $3.1[0.01]$ & $6.1[0.06]$ & $16.9[0.32]$ & $41.8[2.95]$ \\
HbAS-3 & $3.6[0.03]$ & $7.5[0.17]$ & $22.2[0.06]$ & $44.7[1.04]$ \\
\hline HbAS-4 & $4.9[0.03]$ & $8.4[0.00]$ & $23.6[0.10]$ & $47.4[2.35]$ \\
HbAS-5 & $3.9[0.11]$ & $7.4[0.00]$ & $21.2[0.12]$ & $45.8[1.50]$
\end{tabular}

Parasitemia values in percentage average. [StDv]: Standard Deviation among triplicates; h: hours.

During the second proliferative cycle (between 24-48 h post invasion) the emergence of $>4 \mathrm{~N}$ - and the increase of $4 \mathrm{~N}-\mathrm{iRBC}$ revealed the continued cellular proliferation over time. However, unlike the population seen in the control $\mathrm{HbAA}$ cells after $24 \mathrm{~h}$, the parasite population in the HbSS RBC turned structurally stable, with the frequency of subpopulations remaining constant up to the last time point assayed at $72 \mathrm{~h}$. The set of data from four representative HbSS cultures presented in Online Supplementary Figure S1 illustrates that, despite the individual biological features of $\mathrm{RBC}$ from different individuals with SCD, a general common pattern of population structure was adopted by the parasite in sickle cells (HbSS). Once the heterogeneity of stages and sub-parasite populations was achieved at the $24 \mathrm{~h}$ time point in the HbSS cells, the proportion of subpopulations were kept relatively stable throughout the monitored $72 \mathrm{~h}$ period. Comparing these frequencies with those from control HbAA samples showed that the maintenance of relative proportions of sub-populations was not random. Although the parasitemia along with the subpopulation structure varied among the sickle cell anemia samples, the specific proportions of $1 \mathrm{~N}-, 2 \mathrm{~N}-, 4 \mathrm{~N}-$ and $>4 \mathrm{~N}-\mathrm{iRBC}$ were built in the 11 samples that stayed constant from 24-72 h [frequency averages in HbSS iRBC: $1 \mathrm{~N}$ HbSS-iRBC dropped from approx. $68 \%$ at $1 \mathrm{~h}$ to $47 \%$ at $24 \mathrm{~h}$; remained constant throughout $48 \mathrm{~h}$ as $44 \%$ and at $72 \mathrm{~h}$ as $51 \%$; $2 \mathrm{~N}-\mathrm{HbSS}-\mathrm{iRBC}$ from the initial $26 \%$ at $1 \mathrm{~h}$ slightly changed to $28 \%$ up to $72 \mathrm{~h}$; $4 \mathrm{~N}$ reached $20 \%$ at $24 \mathrm{~h}$ and then stayed relatively unchanged up to $48 \mathrm{~h}$ $(21 \%)$ and $16 \%(72 \mathrm{~h}) ;>4 \mathrm{~N}$ reached $7 \%$ at $24 \mathrm{~h}, 10 \%$ at $48 \mathrm{~h}$ and $5 \%$ at $72 \mathrm{~h}$ ] (data not shown). Unlike the HbSS cultures, control HbAA cultures exhibited the typical fluctuating dynamics of parasite culture.

Unlike the parasites in the HbSS cell cultures, parasites grown in wild-type cells (HbAA-RBC) efficiently performed sequential cycles of invasion and egress, as evidenced by the increase in $1 \mathrm{~N}$-iRBC (invasion clearly identified by the arrow between 48-72 h) (Online Supplementary Figure S1A) and the decrease in $4 \mathrm{~N}$ - and $>4 \mathrm{~N}$-iRBC (egress identified by the decrease in quantified frequency $4 \mathrm{~N}$ - and $>4 \mathrm{~N}$-iRBC; data not shown) with a progressive increase in total parasite population size (levels of parasitemia over time HbAA-III; Table 2). This fluctuation among sub-populations of parasites in $\mathrm{HbAA}$ cultures proved that conditions in $\mathrm{HbAA}$ cells were conducive to parasite life-cycle processes that resulted in population growth. Similarly, parasite cultures in HbAS cells exhibited the variation in infected $\mathrm{RBC}$-sub populations expected
Table 4. Inhibition of parasite culture expansion compared to wild-type (HbAA) measured 24-72 hours post invasion in sickle cell anemia (HbSS) and sickle trait (HbAS) from different donors.

$\begin{array}{lccc}\text { Sample } & 24 \mathrm{~h} & 48 \mathrm{~h} & 72 \mathrm{~h} \\ \operatorname{HbSS}(\mathrm{n}=12) & 22[19] & 69[12] & 84[8] \\ \operatorname{HbAS}(\mathrm{n}=6) & 10[8] & 15[11] & 4[4]\end{array}$

Values expressed as average percentage of inhibition compared to control HbAA [StDv]: Standard Deviation among triplicates; h: hours.

over time, based on the sequential process of invasion, development and egress (data not shown). This normal equilibrium among the infected RBC hosting different intra-parasitic loads observed in $\mathrm{HbAA}$ and $\mathrm{HbAS}$ contrasted with the static proportions of the sub-populations among the HbSS cultures (Online Supplementary Figure $S 1 B-E)$ observed after $24 \mathrm{~h}$, and suggested a potential inhibitory environment in the HbSS cell may account for the low parasitemia found in HbSS cultures.

\section{Effect of HbSS cell environment on the morphological differentiation of the parasite}

As shown above, HbSS RBC did not interfere with the intra-erythrocytic parasite proliferation and differentiation since $\mathrm{iRBC}$ were seen carrying high intracellular parasite loads $(4 \mathrm{~N}$ and $>4 \mathrm{~N})$ right from the first $24 \mathrm{~h}$ life cycle (Figure $3 \mathrm{~A}$ ). In addition, the analysis of the sequential appearance of the different morphological stages (light microscopy of Giemsa smears) in the HbAA, HbAS and $\mathrm{HbSS}$ cultures confirmed this successful intracellular proliferation, and showed that parasite differentiation into the different morphological stages occurred successfully in all three types of cultures (Figure 4). However, closer examination of parasite morphology in HbSS cells revealed some abnormalities. The heterogeneity of the sub-populations of iRBC reported from the FACS analysis of these cultures (Figure 3 ) was also observed by microscopic analysis and can be described as a change in frequency of infected RBC hosting different numbers of attached or detached parasites (Figure 4). The predominant parasite stages seen at later time points in sickle cells were of the detached variety and consisted of rings (describes any unattached intracellular parasite) present in single, double, quadruple and multiple forms. Many of the parasites were seen unattached to each other and assumed either circular, pear or deformed shapes (Figure 4). However, there were parasitized RBC that also assumed conventional morphological stages, seen as "paired-fig- 




Figure 3. Distribution of parasite sub-populations cultured in three types of red blood cells (RBC), based on genome content reveals all sub-populations are represented in the cultures. (A) HbSS. (B) HbAS. (C) HbAA. Samples were collected at 1 hour (h), $24 \mathrm{~h}$ and $48 \mathrm{~h}$ and the percentage of each infected RBC sub-population was determined by FACS using VybrantRDyeCycleTMGreen dye to quantify the amount of parasitic DNA within infected cells, where (N) is the number of parasite genomes. (Top left) HbSS cells show higher numbers of $2 \mathrm{~N}$ cells at $1 \mathrm{~h}$ time point compared to (left middle) HbAS and (left bottom) HbAA cells confirming a higher frequency of multiple invasion events in HbSS. (Middle and right panels) Parasite population structure at 24 and $48 \mathrm{~h}$ showing all sub-populations represented in all three cell genotypes.

ures" (2 attached parasites) and/or Maltese Cross (4 attached parasites) and/or double paired-figures (also as 4 parasites attached 2 by 2 ) and were seen co-existing with the detached ring parasites, showing that cellular division and differentiation of $B$. divergens followed the sequential transformation of stages from attached into unattached forms after completing cytokinesis before their egress. In addition, some of the infected RBC in HbSS cells hosted multiple parasites ( $\geq 4-8$ or more) which were detected with variable frequency among HbSS cultures (Figure 4).

Thus, the morphological analysis supported the FACS analysis of population structures at the various sampled time points of the different cultures, HbAA, AS and SS. Importantly, the parasites in the HbSS RBC exhibited features that signaled they were ready for egress, i.e. the presence of both-infected RBC hosting multiple parasites as well as the presence of detached parasites in these cells. However, these parasites were not successful at starting new intra-erythrocytic cycles, as evidenced by the lack of increase in parasitemia.

\section{Parasite infectivity is impaired in HbSS cells as the addition of fresh red blood cells does not rescue parasitemia in HbSS cultures}

Success of the parasite culture propagation as measured by an increase in parasitemia requires successful invasion, successful production and maturation of merozoites which then need to successfully egress. This in turn depends on several factors involving host cells and para- sites. Sickle cell anemia RBC are notorious for exhibiting increased fragility ${ }^{37}$ as compared to HbAA RBC and this could be a factor in the inability of the cultures to support parasitemia after $24 \mathrm{~h}$.

To test whether the lack of increase in parasitemia was a consequence of defective egress, sickle cell fragility or defective merozoite maturation, we examined progression of cultures in which the introduction of fresh $\mathrm{RBC}$ (either $\mathrm{HbAA}$ and $\mathrm{HbSS}$ ) into $24 \mathrm{~h} \mathrm{HbSS}$ parasite cultures was performed. After $1 \mathrm{~h}$, all cells supported Babesia invasion equally well, as shown, following the mixing of merozoite inoculum and RBC (Online Supplementary Table S2). The cultures were then allowed to progress for $24 \mathrm{~h}$ at which point, the parasitemia in both the $\mathrm{HbAA}$ and HbSS cultures had risen slightly (HbAA-A: $3.2 \%$ vs. HbSSA: $3.4 \%$ ) (Online Supplementary Table S2), in line with the results reported for the $12 \mathrm{HbSS}$ cultures above (Tables 1 and 2). The cultures were then split, either staying the same with only medium change (flasks A) or receiving fresh SS cells (flasks B) or AA cells (flasks C), maintaining hematocrits of $5 \%$. At $48 \mathrm{~h}$, the parasitemia in the original HbAA-A culture had doubled (from $3.2 \%$ to approx. $6.5 \%$ ) while that of the original HbSS-A culture reported only a small increase in parasitemia (from 3.4\% to approx. $4 \%$ ), following the pattern reported for the original 12 HbSS cultures (Tables 1 and 2). The HbAA-B culture which was diluted with fresh HbAA RBC to receive half of the inoculum exhibited half the parasitemia of the undiluted HbAA-A culture, once again as expected (3.2\%) 
(Online Supplementary Table S2). The two HbSS cultures that received fresh $\mathrm{RBC}$, either $\mathrm{HbAA}(\mathrm{HbSS}-\mathrm{C})$ or $\mathrm{HbSS}$ (HbSS-B), had lower parasitemias $(2.1 \%$ and $2.5 \%$, respectively; $24 \mathrm{~h}$ time point) than that of $\mathrm{HbAA}-\mathrm{B}$ culture, proving that the nature or condition of the host cells did not play a role in the inhibition of new cycles of parasitemia seen in the HbSS cultures (HbSS-C and HbSS-B). At later time points ( $48 \mathrm{~h}$ after addition of fresh cells), this result of non-rescue was further strengthened when cultures in HBAA-B exhibited a robust increase in parasitemia (16\%), contrasting with HbSS-B and HbSS-C and the stable parasitemia of ( $3.6 \%$ and $2.3 \%$, respectively). The original half culture which had only medium change (HbAA-A and HbSS-A) followed the previously reported inhibition patterns of in culture progression showing approximately 18\% inhibition in HbSS-A culture growth, compared to the HbAA-A culture. Neither the addition of fresh $\mathrm{HbSS} \mathrm{RBC}$ or $\mathrm{HbAA} \mathrm{RBC}$ could significantly rescue the HbSS cultures, with the original HbSS-A parent culture at $4.7 \%$ and the culture receiving fresh HbSS cells at $3.6 \%$ parasitemia. Surprisingly, the culture that received fresh $\mathrm{HbAA}$ cells was even lower demonstrating an infection rate of $2.3 \%$, indicating that parasite development within the HbSS RBC was potentially impaired, resulting in the formation of merozoites unable to invade fresh $\mathrm{RBC}$ at frequencies typically seen in vitro parasite cultures. The formation of mature merozoites that egress from the $\mathrm{RBC}$ are critical to start new intra-erythrocytic cycles by invading new host cells, and these merozoites are required to be in an optimum invasive state to support new cycles.

\section{Parasite egress from sickle red blood cells may also be compromised}

One of the factors that may impact the progress in parasitemia in HbSS cells is the ability of the parasite to egress from the host sickle cell and invade new host cells. The FACS profile of the parasite population in the HbSS cells was carefully examined to look for this inhibition of egress. The typical pattern of parasite-holding within the $\mathrm{RBC}$ that we have reported for $B$. divergens, ${ }^{31}$ when there is an egress defect, where the $B$. divergens parasite population builds its $4 \mathrm{~N}$ and $>4 \mathrm{~N}$ populations to high levels that
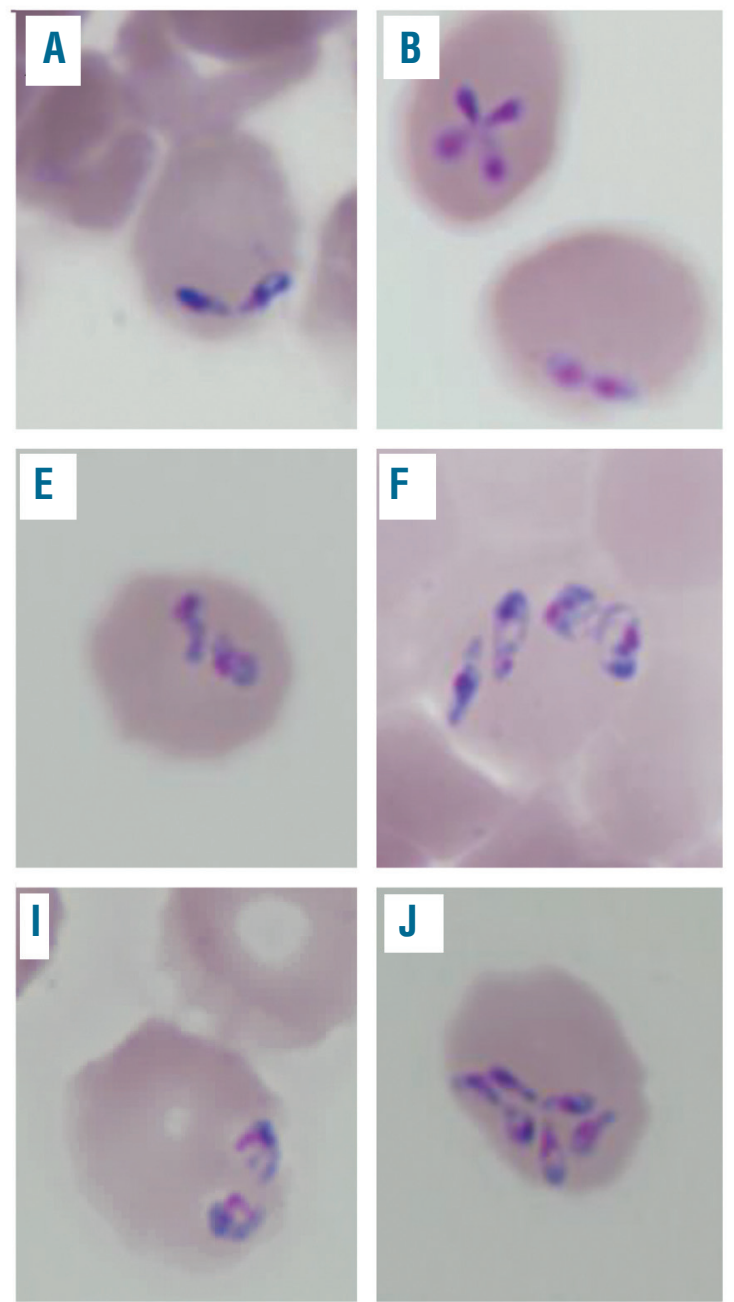
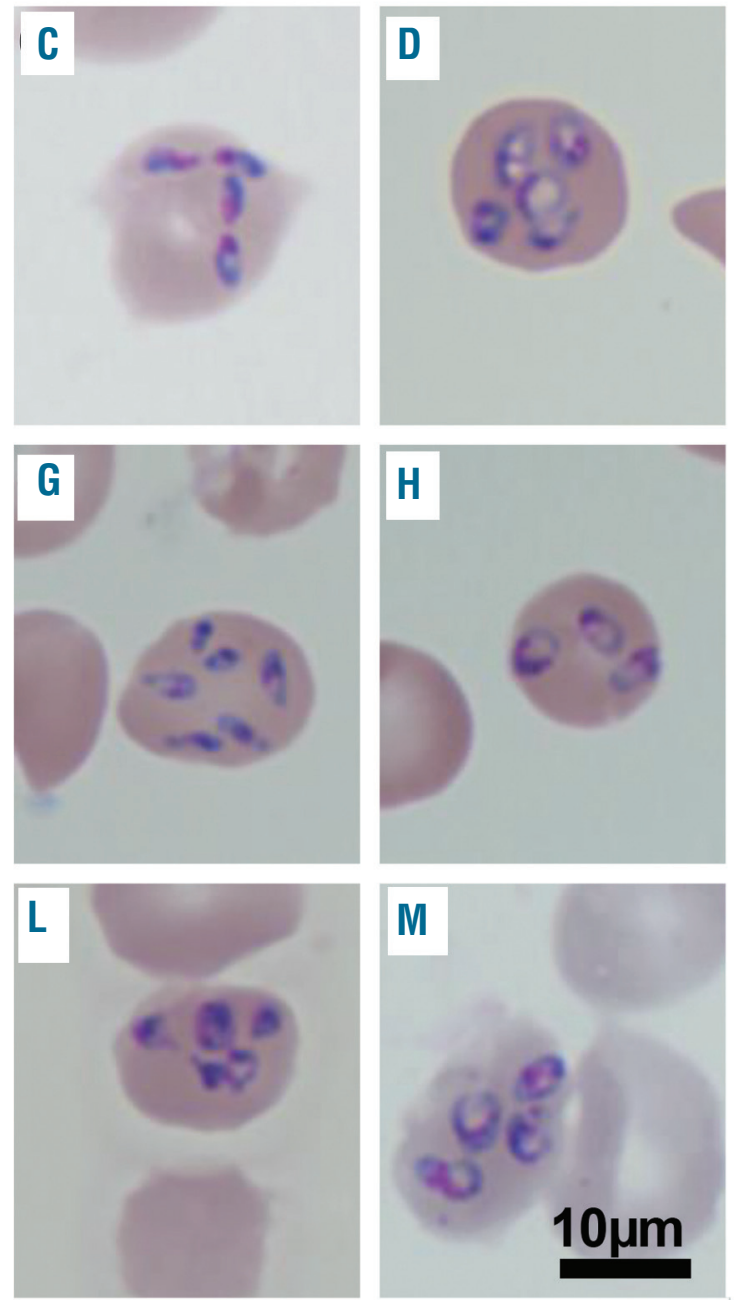

Figure 4. Parasites grown in HbSS cells exhibit atypical morphology although normal parasite forms are also seen in the same cultures. (A-C) Normal morphology of parasite seen as paired figures or Maltese Crosses or double paired figures (D-M) Unusually high numbers of detached rings seen at high frequency in all HbSS parasite cultures. 
can be visualized by both FACS analysis and Giemsa stained smears, was not seen in these sickle cell cultures (Figures 3 and 4). In fact, the presence of free merozoites was noted in most culture supernatants (data not shown), although it is difficult to quantitatively estimate if these numbers are normal as compared to wild-type RBC. However, unlike the fluctuating proportions of the parasite sub-populations seen in both $\mathrm{HbAA}$ and $\mathrm{HbAS}$ cultures, relatively stable proportions of parasite sub-populations in the sickle RBC cultures, after the $24 \mathrm{~h}$ time point was a characteristic noted in all $12 \mathrm{HbSS}$ cultures (Online Supplementary Figure S1). This novel holding pattern could potentially signify an inability of the parasites to egress, which in turn could cause the relatively static parasitemia observed in these cultures. Thus, inefficient parasite egress from the host HbSS cells infected in the first round of invasion may not permit new cycles of invasion to take place.

\section{Discussion}

Variant RBC are produced from some of the most common human genetic polymorphisms, and their high incidence has been ascribed to the evolutionary selection by life-threatening falciparum malaria. ${ }^{38,39}$ The sickle hemoglobin genotype (HbAS/HbSS) is the best-characterized human genetic polymorphism associated with malaria. In this paper, we have explored the effect of the sickle hemoglobin mutation on a related intra-erythrocytic apicomplexan parasite, $B$. divergens. There are several stages in the parasite's erythrocytic cycle at which RBC mutations can affect parasite infection. The first would be erythrocyte invasion by Babesia merozoites; the next stage susceptible to inhibition is the intra-erythrocytic parasite development. This category would include impairments in the parasite's ability to meet its nutritional requirements or changes in the host cell milieu that would be cidal to the parasite. Finally, impairment of red cell rupture and release of infective merozoites at parasite maturity could inhibit increase in infection. Use of in vitro growth assays as our primary outcome, along with a robust sample size of SCD $\mathrm{RBC}$, provided a rare opportunity to systematically examine the cellular determinants of parasite growth in the sickle setting. We present here evidence for atypical population progression, a potential loss of merozoite infectivity, and defective egress of the parasite in these hemoglobinopathic cells (Tables 1-4 and Online Supplementary Table S2). Interestingly, using the Townes mouse model of SCD, and $B$. microti, we have shown that a similar inhibition results in dramatically low infection rates in $\mathrm{HbSS}$ mice as compared to HbAS and HBAA mice. ${ }^{40}$

Invasion of the human RBC is the central pathogenic step in the life-cycle of Babesia. When Babesia spp. sporozoites are first injected into the human host with a tick bite, they target the host RBC immediately, unlike Plasmodium spp. which are required to undergo an exo-erythrocytic phase in hepatic cells. It is the parasite's ability to first recognize and then invade host $\mathrm{RBC}$ that is central to symptomatic human babesiosis, and the parasites invade RBC using multiple complex interactions between parasite proteins and the host cell surface, which have not been fully elucidated. ${ }^{2,41,42}$ Like Plasmodium, $B$. divergens has been shown to use GPA and GPB as invasion receptors. ${ }^{33}$ In vitro studies with $P$. falciparum have suggested a decreased invasion and growth of the parasite in sickle cells; ${ }^{43-45}$ however, some of the older studies have not been able to differentiate between the various phases of the parasite lifecycle. Hence, a deficiency in egress or invasion would both be visualized as an overall decrease in parasitemia. The use of our platform combining in vitro invasion and growth assays with synchronized cultures monitored by both FACS analysis and Giemsa smears provided an excellent system to systematically dissect the phase of the erythrocytic cycle impacted by the HbSS environment. The increased DNA content of HbSS cells that contributed to the subtle higher invasion percentages was found to be due to the presence of the Howell-Jolly bodies which artefactually increased the DNA load of the cell. When the contribution of the bodies was subtracted, a similar rate of invasion was obtained in all $\mathrm{Hb}$ genotypes, indicating a potential difference between malaria and Babesia mechanisms of invasion in sickle cells.

The development of the parasite in HbSS cells in the first $24 \mathrm{~h}$ was normal and exhibited all conventional forms reported in vitro cultures by us earlier. ${ }^{34}$ Thus, rings, paired figure and Maltese Cross forms were all documented in culture. However, a larger than expected proportion of cells hosted detached rings (Figure 4). The multiple unattached parasites feature assumed by parasites in $\mathrm{HbSS}$ cultures suggests that the parasite is able to complete cytokinesis shortly after the nuclear duplication, allowing the conclusion of cell division which may not allow the accumulation of attached morphological stages such as Maltese-Cross or paired-figures, as seen in HbAA cultures. After $24 \mathrm{~h}$, the progression of cultures is stalled in HbSS cells as seen by FACS analysis (Figure $3 \mathrm{~A}$ and Online Supplementary Figure $S 1 B-E$ ) where the sub-populations remain in static proportions unlike the dynamic movements seen among sub-populations in $\mathrm{HbAA}$ (Figure $3 \mathrm{C}$ and Online Supplementary Figure S1A) and AS cells (Figure $3 \mathrm{~B})$. As this profile is representative of the parasite population and not individual parasites, it is apparent that, overall, there is no growth in the population despite small increases seen in parasitemia, reflecting a minority of parasites successfully initiating new cycles of infection. Microscopic analysis of the parasites in HbSS cells reveals normal morphology in terms of size, shape and staining patterns. Ultra-structural analysis may shed more light on potential defects in these parasites, if present.

Egress is a phase of the cycle that, if impacted, can lead to disastrous outcomes for the parasite population progression. In vitro studies with $P$. falciparum suggest a link between the hydration status of the host $\mathrm{RBC}$ and parasite invasion and egress. ${ }^{46,47}$ The high water-permeability of the RBC ensures their continued osmotic equilibrium in plasma so that they can shrink or swell by the loss or gain of a fluid isosmotic with surrounding plasma. This homeostatic balance is disrupted in $\mathrm{HbSS}$ cells, resulting in altered ion fluxes, ion content regulation, and hydration states in the circulation. ${ }^{48}$ Malaria parasites have to breach both the parasitophorous vacuolar membrane (PVM) and erythrocyte membrane in order to egress. The altered exit of malaria merozoites from the dehydrated RBC was linked to the reduction of osmotic pressure within the parasitophorous vacuole that was needed to lyse the compartment prior to lysis of the RBC membrane. However, intra-erythrocytic Babesia parasites are free in the cytoplasm of the RBC without being enclosed in a vacuole as the PVM is a transient structure found fleetingly after 
invasion of the Babesia merozoite. Thus, the lack of osmotic pressure in the infected sickle RBC may not impact Babesia parasite egress. Additionally, FACS analysis of the cultures at later time points do not support the typical loss of egress phenotype, which presents as a build-up of $4 \mathrm{~N}$ and $>4 \mathrm{~N}$ populations in a single cell, as seen in our previous work with egress inhibitors. ${ }^{31}$ Although there were cells that hosted multiple parasites $(>4 \mathrm{~N})$, these appeared to be a result of the normal proliferative cycle to build the population structure, but they were not the majority of infected cells. However, the novel holding pattern reported here (Online Supplementary Figure S1), where the sub-populations are maintained after $24 \mathrm{~h}$ in the same ratios, indicate that egress may be compromised in these cells. Thus, it is possible that HbSS cells were not as efficient in supporting egress as HbAA cells, but other factors also contribute to the low infection rates seen in these cultures. Production of viable, infective merozoites within HbSS cell appeared to be another limitation of these cultures. Although free merozoites were seen in most HbSS culture supernatants, they did not appear to be able to successfully initiate new rounds of infection. Rescue experiments with both fresh HbSS and HbAA cells indicate that the defect may not lie in the unavailability of optimal host cells but rather with the merozoite, despite them presenting normal morphology (Online Supplementary Table S2). Detailed morphological examination of these zoites by electron microscopy may provide clues to their lack of infectivity.

Despite years of research effort, the mechanism of protection of the sickle trait in malaria remains unclear. A number of cellular, biochemical and immune-mediated mechanisms have been proposed, and it is likely that multiple complex mechanisms are responsible for the observed protection. Invasion and growth of $P$. falciparum in vitro within $\mathrm{HbAS} \mathrm{RBC}$ is reduced in low oxygen tension growth conditions $\left(<5 \% \mathrm{O}_{2}\right) .{ }^{43,49}$ Some have proposed that the increased sickling of infected $\mathrm{HbAS} \mathrm{RBC}$, due to polymerization of sickle $\mathrm{Hb}$, may be a mechanism for impaired growth under low $\mathrm{O}_{2}$ conditions. ${ }^{50,51}$ However, in our in vitro assays, which utilize micro-aerophilus conditions with $5 \% \mathrm{O}_{2}$, no inhibition of Babesia growth was observed in the HbAS cells, in which all five samples yielded comparable support of parasite growth as that seen in control HbAA RBC. Unlike Plasmodium, which digests $\mathrm{Hb}$ to meet its nutritional needs, Babesia does not digest any of the $\mathrm{Hb}$, which may account for the lack of inhibition of intra-erythrocytic proliferation seen in the $\mathrm{HbAS}$ cells (no significant difference between $\mathrm{HbAA}$ and HbAS cells; $P>0.05$ ) (Table 3 ). As the assays reported here are purely in vitro assays, the effect of in vivo parameters like increased cytoadhesion, ${ }^{52}$ splenic retention, ${ }^{53}$ and altered immune system response, as seen in enhanced phagocytosis and induction of inflammatory cytokines following endothelial activation, are not factored in, which may lead to an overall protection against the parasite in vivo, as seen in malaria. Future studies documenting the incidence of babesiosis in the sickle cell trait population compared to that in the HbAA individuals will confirm these findings.

In this paper, we present evidence for altered parasite population progression caused potentially by defective merozoite maturation and/or defective egress from the sickle cell anemia RBC. The latter two phenomena when present alone or together may explain the inability of $\mathrm{HbSS}$ cells to support high infection rates in vitro. It is not clear whether the parasite would behave similarly in vivo. We have recently shown in a mouse SCD model similar inhibition of parasite population progression, and the results presented in this study confirm the inability of the Babesia parasite to thrive in the sickle cell anemia setting. ${ }^{40}$ In a significant contrast to malaria, sickle trait cells seemed to sustain parasite infection rates comparable to those of wild-type RBC, and, once again, these in vitro studies need to be supplemented by comparable in vivo analyses under conditions of differing oxygen tension, which has been shown to play a role in suppressing malaria parasitemia. Patients with SCD are on chronic transfusion therapy and may develop severe transfusion-associated Babesia infection. ${ }^{29}$ Studies dissecting the cellular and molecular mechanisms of parasite growth within sickle cell patients that may impact the pathogenesis of babesiosis in sickle cell and other hemoglobinopathies are needed to define potential novel therapies against this disease.

\section{Acknowledgments}

We thank Vijay Nandi, PhD (Laboratory Data Analytic Services, NYBC) for help with the statistical analysis.

\section{Funding}

This research was funded by NIH grant to CL-HL140625 and Hugoton Foundation grant to JRCS-HUG 575.

\section{References}

1. Lelliott PM, McMorran BJ, Foote SJ, Burgio $G$. The influence of host genetics on erythrocytes and malaria infection: is there therapeutic potential? Malar J. 2015;14:289.

2. Ord RL, Lobo CA. Human Babesiosis: Pathogens, Prevalence, Diagnosis and Treatment. Curr Clin Microbiol Rep. 2015;2(4):173-181

3. Alaganan A, Singh P, Chitnis CE. Molecular mechanisms that mediate invasion and egress of malaria parasites from red blood cells. Curr Opin Hematol. 2017;24(3):208214.

4. Taylor SM, Fairhurst RM. Malaria parasites and red cell variants: when a house is not a home. Curr Opin Hematol. 2014;21(3):193 200.

5. Goheen MM, Campino S, Cerami C. The role of the red blood cell in host defence against falciparum malaria: an expanding repertoire of evolutionary alterations. $\mathrm{Br} \mathrm{J}$ Haematol. 2017;179(4):543-556.

6. Williams TN. Human red blood cell polymorphisms and malaria. Curr Opin Microbiol. 2006;9(4):388-394.

7. Kwiatkowski DP, Luoni G. Host genetic factors in resistance and susceptibility to malaria. Parassitologia. 2006;48(4):450-467.

8. Weatherall DJ, Provan AB. Red cells I: inherited anaemias. Lancet. 2000;355 (9210):1169-1175.

9. Taylor SM, Cerami C, Fairhurst RM.
Hemoglobinopathies: slicing the Gordian knot of Plasmodium falciparum malaria pathogenesis. PLoS Pathog. 2013;9(5): e1003327.

10. May J, Evans JA, Timmann C, et al Hemoglobin variants and disease manifestations in severe falciparum malaria. JAMA 2007;297(20):2220-2226.

11. Taylor SM, Parobek CM, Fairhurst RM Haemoglobinopathies and the clinical epidemiology of malaria: a systematic review and meta-analysis. Lancet Infect Dis. 2012;12(6):457-468

12. Ingram VM. Abnormal human haemoglobins. III. The chemical difference between normal and sickle cell haemoglobins. Biochim Biophys Acta. 1959;36:402-411. 
13. Macharia AW, Mochamah G, Uyoga S, et al. The clinical epidemiology of sickle cell anemia In Africa. Am J Hematol. 2018;93(3):363-370

14. Aidoo M, Terlouw DJ, Kolczak MS, et al. Protective effects of the sickle cell gene against malaria morbidity and mortality. Lancet. 2002;359(9314):1311-1312.

15. Gong L, Parikh S, Rosenthal PJ, Greenhouse B. Biochemical and immunological mechanisms by which sickle cell trait protects against malaria. Malar J. 2013;12:317.

16. Beaudry JT, Krause MA, Diakite SA, et al. Ex-vivo cytoadherence phenotypes of Plasmodium falciparum strains from Malian children with hemoglobins A, S, and C. PloS One. 2014;9(3):e92185.

17. Western KA, Benson GD, Gleason NN, Healy GR, Schultz MG. Babesiosis in a Massachusetts resident. N Engl J Med. 1970;283(16):854-856.

18. Skrabalo Z, Deanovic Z. Piroplasmosis in man; report of a case. Doc Med Geogr Trop. 1957;9(1):11-16

19. Bloch EM, Herwaldt BL, Leiby DA, et al. The third described case of transfusiontransmitted Babesia duncani. Transfusion. 2012;52(7):1517-1522.

20. Conrad PA, Kjemtrup AM, Carreno RA, et al. Description of Babesia duncani n.sp. (Apicomplexa: Babesiidae) from humans and its differentiation from other piroplasms. Int J Parasitol. 2006;36(7):779-789.

21. Jiang JF, Zheng YC, Jiang RR, et al. Epidemiological, clinical, and laboratory characteristics of 48 cases of "Babesia venatorum" infection in China: a descriptive study. Lancet Infect Dis. 2015;15(2):196-203

22. Sun Y, Li SG, Jiang JF, et al. Babesia venatorum infection in child, China. Emerg Infect Dis. 2014;20(5):896-897.

23. Haselbarth K, Tenter AM, Brade V, Krieger $G$, Hunfeld KP. First case of human babesiosis in Germany - Clinical presentation and molecular characterisation of the pathogen. Int J Med Microbiol. 2007;297(3):197-204.

24. Herwaldt BL, Caccio S, Gherlinzoni F, et al. Molecular characterization of a non-Babesia divergens organism causing zoonotic babesiosis in Europe. Emerg Infect Dis. 2003;9(8):942-948

25. Yabsley MJ, Shock BC. Natural history of Zoonotic Babesia: Role of wildlife reservoirs. Int J Parasitol Parasites Wildl. 2013; 2:18-31

26. Gubernot DM, Nakhasi HL, Mied PA, Asher DM, Epstein JS, Kumar S. Transfusion-transmitted babesiosis in the United States: summary of a workshop. Transfusion. 2009;49(12):2759-2771.

27. Leiby DA. Transfusion-associated babesiosis: shouldn't we be ticked off? Ann Intern
Med. 2011:155(8):556-557.

28. Lobo CA, Cursino-Santos JR, Alhassan A, Rodrigues M. Babesia: an emerging infectious threat in transfusion medicine. PLoS Pathog. 2013;9(7):e1003387.

29. Karkoska K, Louie J, Appiah-Kubi AO, et al. Transfusion-transmitted babesiosis leading to severe hemolysis in two patients with sickle cell anemia. Pediatr Blood Cancer 2018;65(1)

30. Cushing M, Shaz B. Transfusion-transmitted babesiosis: achieving successful mitigation while balancing cost and donor loss. Transfusion. 2012;52(7):1404-1407.

31. Cursino-Santos JR, Singh M, Pham P, Lobo CA. A novel flow cytometric application discriminates among the effects of chemical inhibitors on various phases of Babesia divergens intraerythrocytic cycle. Cytometry A. 2017;91(3):216-231

32. Gorenflot A, Brasseur P, Precigout E L'Hostis M, Marchand A, Schrevel J. Cytological and immunological responses to Babesia divergens in different hosts: ox, gerbil, man. Parasitol Res. 1991;77(1):3-12.

33. Lobo CA. Babesia divergens and Plasmodium falciparum use common receptors, glycophorins A and B, to invade the human red blood cell. Infect Immun. 2005; 73(1):649-651.

34. Cursino-Santos JR, Singh M, Pham P, Rodriguez M, Lobo CA. Babesia divergens builds a complex population structure composed of specific ratios of infected cells to ensure a prompt response to changing environmental conditions. Cell Microbiol. 2016;18(6):859-874.

35. Harrod VL, Howard TA, Zimmerman SA, Dertinger SD, Ware RE. Quantitative analysis of Howell-Jolly bodies in children with sickle cell disease. Exp Hematol. 2007; 35(2):179-183.

36. El Hoss S, Dussiot M, Renaud O, Brousse V, El Nemer W. A novel non-invasive method to measure splenic filtration function in humans. Haematologica. 2018; 103(10):e436-e439.

37. Rees DC, Williams TN, Gladwin MT. Sickle-cell disease. Lancet. 2010; 376(9757):2018-2031

38. Bunn HF. The triumph of good over evil: protection by the sickle gene against malaria. Blood. 2013;121(1):20-25

39. Elguero E, Delicat-Loembet LM, Rougeron $V$, et al. Malaria continues to select for sickle cell trait in Central Africa. Proc Natl Acad Sci U S A. 2015;112(22):7051-7054

40. Yi W, Bao W, Rodriguez M, et al. Robust adaptive immune response against Babesia microti infection marked by low parasitemia in a murine model of sickle cell disease. Blood Adv. 2018;2(23):3462-3478.
41. Lobo CA, Rodriguez M, Cursino-Santos JR Babesia and red cell invasion. Curr Opin Hematol. 2012;19(3):170-175.

42. Cursino-Santos JR, Halverson G, Rodriguez M, Narla M, Lobo CA. Identification of binding domains on red blood cell glycophorins for Babesia divergens. Transfusion. 2014;54(4):982-989.

43. Friedman MJ. Erythrocytic mechanism of sickle cell resistance to malaria. Proc Nat Acad Sci U S A. 1978;75(4):1994-1997.

44. Pasvol G, Weatherall DJ, Wilson RJ. Cellular mechanism for the protective effect of haemoglobin $\mathrm{S}$ against $P$. falciparum malaria. Nature. 1978; 274 (5672):701-703.

45. Pasvol G. The interaction between sickle haemoglobin and the malarial parasite Plasmodium falciparum. Trans R Soc Trop Med Hyg. 1980;74(6):701-705.

46. Tiffert T, Lew VL, Ginsburg H, Krugliak M Croisille L, Mohandas N. The hydration state of human red blood cells and their susceptibility to invasion by Plasmodium falciparum. Blood. 2005;105(12):4853-4860.

47. Glushakova S, Humphrey G, Leikina E, Balaban A, Miller J, Zimmerberg J. New stages in the program of malaria parasite egress imaged in normal and sickle erythrocytes. Curr Biol. 2010;20(12):1117-1121.

48. Lew VL, Bookchin RM. Ion transport pathology in the mechanism of sickle cell dehydration. Physiol Rev. 2005;85(1):179 200.

49. Goheen MM, Wegmuller R, Bah A, et al Anemia Offers Stronger Protection Than Sickle Cell Trait Against the Erythrocytic Stage of Falciparum Malaria and This Protection Is Reversed by Iron Supplementation. EBioMedicine. 2016; 14:123-130

50. Luzzatto L, Nwachuku-Jarrett ES, Reddy S. Increased sickling of parasitised erythrocytes as mechanism of resistance against malaria in the sickle-cell trait. Lancet. 1970; 1(7642):319-321.

51. Archer NM, Petersen N, Clark MA, Buckee CO, Childs LM, Duraisingh MT. Resistance to Plasmodium falciparum in sickle cell trait erythrocytes is driven by oxygen-dependent growth inhibition. Proc Natl Acad Sci U S A. 2018:115(28):7350-7355.

52. Cholera R, Brittain NJ, Gillrie MR, et al Impaired cytoadherence of Plasmodium falciparum-infected erythrocytes containing sickle hemoglobin. Proc Natl Acad Sci U S A. 2008;105(3):991-996.

53. Diakite SA, Ndour PA, Brousse V, et al. Stage-dependent fate of Plasmodium falciparum-infected red blood cells in the spleen and sickle-cell trait-related protection against malaria. Malar J. 2016;15(1):482. 